\title{
Astrophysical structures from primordial quantum black holes
}

\author{
Salvatore Capozziello ${ }^{1,2}$, Gerardo Cristofano ${ }^{1,2}$, Mariafelicia De Laurentis ${ }^{1,2}$ \\ ${ }^{1}$ Dipartimento di Scienze Fisiche, Università di Napoli "Federico II", ${ }^{2}$ INFN Sez. di Napoli, \\ Compl. Univ. di Monte S. Angelo, Edificio G, Via Cinthia, I-80126, Napoli, Italy
}

(Dated: November 11, 2018)

\begin{abstract}
The characteristic sizes of astrophysical structures, up to the whole observed Universe, can be recovered, in principle, assuming that gravity is the overall interaction assembling systems starting from microscopic scales, whose order of magnitude is ruled by the Planck length and the related Compton wavelength. This result agrees with the absence of screening mechanisms for the gravitational interaction and could be connected to the presence of Yukawa corrections in the Newtonian potential which introduce typical interaction lengths. This result directly comes out from quantization of primordial black holes and then characteristic interaction lengths directly emerge from quantum field theory.
\end{abstract}

PACS numbers: $04.50 .+\mathrm{h}, 95.36 .+\mathrm{x}, 98.80 .-\mathrm{k}$

Keywords: Alternative theories of gravity; cosmology; conformal invariance; quantum field theory

\section{INTRODUCTION}

Framing the astrophysical, self-gravitating structures in a self-consistent scheme is one of the main puzzles of modern physics since the growing amount of observations seems to escape any coherent interpretative scheme. Essentially, from the fundamental physics point of view, we would like to re-conduct cosmic structures and their evolution to some unifying theory in which all the today observed interactions are treated under the same standard. In such a scheme, what we observe today on cosmological and astrophysical scales would be just a consequence of quantum fluctuations in early epochs. Then, we should seek for some dynamical mechanism that, after one (or more than one) symmetry breaking, would be capable of yielding structures like clusters of galaxies, galaxies and then stars from primordial quantum spectra of perturbations.

The so called "inflationary paradigm" [1] related to several unifying theories (e.g. superstrings, GUT, SUSY) seems adequate to this goal if one were able to definitely select the right model.

On the other hand, particle physics needs cosmological predictions and observations since the energies for testing unified theories are so high that it is extremely unlikely that they will be ever reached on Earth-based laboratories. As a matter of fact, cosmology needs particle physics and vice versa. The point is that remnants of primordial epochs should be found by cosmological observations and, by them, one should constrain elementary particle physics models ${ }^{1}$.

This philosophy has been pursued by several researchers; first of all by Sakharov [2] who in 1965 ar-

\footnotetext{
1 Results of Large Hadron Collider (LHC) at CERN could be the "experimentum crucis" since, due to the energies involved, their outcome should be related to the thermodynamical state of primordial Universe.
}

gued that quantum primordial fluctuations should have expanded towards the present epoch leading first to classical energy-density perturbations and, after the decoupling from the cosmological background, to the observed galaxies, clusters, super-clusters of galaxies, and afterwards stars. Shortly, the underlying issue of any modern theory of cosmological perturbation is this: primordial quantum fluctuations should be enlarged by cosmological dynamics to the present large scale structures. Now the problem is not only whether observations agree with this scheme (e.g. COBE, IRAS, WMAP, PLANCK data or large scale structure surveys [3] ) but, mainly, whether the astrophysical and cosmological systems "remember" their quantum origin or not.

Despite of the apparent sharp division of the classical and quantum worlds, macroscopic quantum phenomena exist and some behaviors of classical systems can be explained only in the framework of quantum mechanics. The high $T_{c}$ superconductivity and several other macroscopic coherent systems are interesting instances of these peculiar phenomena in which a quantum "memory" persists at the macroscopic scale.

Some intriguing conjectures have been proposed to find quantum signatures at the classical, macroscopic scales of astrophysical structures [4]. In this scheme, classical laws of force describing the interactions among the constituents of $N$-particle systems of mean length scale $R$, lead to a quantum characteristic action per particle. The forces considered can be, for instance, the electromagnetic interactions between charged particles in large macroscopic systems as charged beams in particle accelerators, plasmas, and neutral dipolar crystals, or the strong interactions between quarks in hadronic bound aggregates, and so on.

Having such a procedure at hand, it seems very natural to investigate whether it can be applied to determine the existence of quantum signatures or "memories" for astrophysical structures.

It can be shown that the order of magnitude of characteristic observed radii of typical galaxies and large scale 
structures can be inferred starting from the microscopic fundamental scales through a phenomenological scaling law [5, 6]. Further scaling relations can be derived, showing that such a connection can be equivalently obtained either by considering the nucleons as the elementary constituents of a galaxy, or, as usual in astrophysics, by considering stars as its granular components. The key issue is that we can define an interaction length $\lambda$ and a typical number of elementary constituents $N$ (e.g. $N_{n}$ the number of nucleons with $\lambda_{c}$, the Compton wavelength, or $N_{s}$ the number of stars with $\lambda_{s}$, the typical interaction length of a star, for example the "size" of the Solar System) and, by the relation

$$
R \simeq \lambda \sqrt{N},
$$

to obtain the observed typical size (in the case of galaxies we obtain $\sim 10 \mathrm{kpc}$ a typical galactic scale).

In the present paper, we show that such a relation holds for all self-gravitating systems as stellar globular clusters, clusters and super-clusters of galaxies and we show that it can be implemented by taking into account a gravitational interaction, explicitly scale-dependent, as predicted by quantum field theory in the low energy limit and resulting, at macroscopic level, as an extended theory of gravity where more general actions of curvature invariants than that by Hilbert and Einstein are considered.

More precisely, renormalizable quantum fields in a gravitational background give rise to modifications, in the low energy limit, of the Newtonian potential. Furthermore, if we do not require enormous amounts of dark matter as the only mechanism to explain the puzzle of the present day astrophysical observations, a scaledependent gravitational interaction is needed. This point of view is supported by several authors [8] which state that recent experiments on cosmic microwave background as BOOMERANG, WMAP and PLANCK [9] or astrophysical structures can be explained by taking into account modified Newtonian dynamics [10].

The present paper is organized as follows. Sec. II is devoted to the discussion of stationary points emerging from quantum field theory in curved space-time where Schwarzschild radius and fundamental electric charge are considered. In Sec. III, we discuss the Lagrangian approach to the so called "attractor mechanism" while, in Sec. IV, we deal with the emergence of quantum charged black holes which could give rise, in principle, to the fundamental scale ruling all the further, larger astrophysical scales. In Sec. V, we show that the weak field limit of extended theories of gravity, coming from quantum field theories formulated in curved space-times, gives rise to Yukawa-like corrections to the Newtonian potential, which, naturally imply characteristic lengths. Sec. VI is devoted to the discussion of characteristic sizes of astrophysical structures. In Sec. VII, we discuss how time-statistical fluctuations of granular components of self-gravitating systems are related to their characteristic sizes. Conclusions are drawn in Sec. VIII.

\section{CHARACTERISTIC LENGTHS FROM QUANTUM FIELD THEORY}

Let us start our considerations from a ReissnerNordstrom metric in order to identify a space-time with a unitary electric charge and a mass acting as source of gravitational field. It is [1]

$$
c^{2} d t^{2}=\left(1-\frac{r_{S}}{r}+\frac{r_{Q}}{r^{2}}\right) c^{2} d t^{2}-\frac{d r^{2}}{1-\frac{r_{S}}{r}+\frac{r_{Q}^{2}}{r^{2}}}-r^{2} d \Omega^{2},
$$

where $d \Omega=d \theta^{2}+\sin ^{2} \theta d \phi^{2}$ is the solid angle, $r_{S}$ is Schwarzschild radius of the massive body $r_{S}=\frac{2 G M}{c^{2}}$ where $G$ is the gravitational constant, and $r_{Q}$ is a lengthscale related to the electric charge $Q r_{Q}^{2}=\frac{Q^{2} G}{4 \pi \epsilon_{0} c^{4}}$, where $\frac{1}{4 \pi \epsilon_{0}}$ is the Coulomb constant. In the limit where the charge $Q$ (or equivalently, the length-scale $r_{Q}$ ) goes to zero, one recovers the Schwarzschild metric. The classical Newtonian theory of gravity is recovered in the limit $\frac{r_{S}}{r} \rightarrow 0$. In this limit, the Minkowski metric

$$
c^{2} d \tau^{2}=c^{2} d t^{2}-d r^{2}-r^{2} d \Omega^{2}
$$

is recovered. Although charged black holes with $r_{Q}<<$ $r_{S}$ are similar to the Schwarzschild black hole, they have two horizons: the event horizon and an internal Cauchy horizon. As usual, the event horizon can be reliably identified by analyzing the equation

$$
g_{00}=1-\frac{r_{S}}{r}+\frac{r_{Q}}{r^{2}}=0 .
$$

This quadratic equation for $r$ has the solutions

$$
r_{ \pm}=\left(\frac{\sqrt{G}}{c^{2}}\right)\left(\frac{r_{S} \pm \sqrt{r_{S}^{2}-4 r_{Q}^{2}}}{r}\right)
$$

These concentric event horizons become degenerate for $2 r_{Q}=r_{S}$ which corresponds to an extremal black hole ${ }^{2}$. That is

$$
\frac{2 G M}{c^{2}}=\frac{2 Q \sqrt{G}}{c^{2} \sqrt{4 \pi \epsilon_{0}}}
$$

\footnotetext{
2 Black holes with $2 r_{Q}>r_{S}$ are believed not to exist in nature because they would contain a naked singularity; their appearance would contradict cosmic censorship hypothesis which is generally believed to hold [12]
} 
or better squaring both members get

$$
\begin{aligned}
\frac{4 G^{2} M^{2}}{c^{4}} & =\frac{2 Q}{4 \pi \epsilon_{0}} \frac{G}{c^{4}}, \\
G M^{2} & =\frac{Q^{2}}{4 \pi \epsilon_{0}} .
\end{aligned}
$$

If the extreme black hole has also a magnetic charge $m$ then

$$
r_{Q}^{2} \rightarrow r_{Q}^{2}+r_{m}^{2}=\frac{Q^{2}}{4 \pi \epsilon_{0}} \frac{G}{c^{4}}+m^{2} \frac{\mu_{0}}{4 \pi} \frac{G}{c^{4}}
$$

and going through the same steps, we get

$$
G M^{2}=\frac{Q^{2}}{4 \pi \epsilon_{0}} \frac{G}{c^{4}}+m^{2} \frac{\mu_{0}}{4 \pi} \frac{G}{c^{4}},
$$

or, in Planck units ${ }^{3}$,

$$
M^{2}=Q_{e}^{2}+Q_{m}^{2}
$$

after using the more convenient notations $Q_{e}=Q$ and $Q_{m}=m$ for the electric and magnetic charges. Some comments are in order at this point.

- Eq.10) represents a no-net force condition: black holes appear to be like dyons that are objects with mass $M$, electric charge $Q_{e}$ and magnetic charge $Q_{m}$. Then the above relation tells that the attractive gravitational force between such two objects at distance $r$ compensates the repulsive electric and magnetic forces between them.

- Eq.10 expresses the saturation of the so called Bogomol'nyi-Prasad-Sommerfeld (BPS) bound (see [13]) expressing the requirement that the local charge density does not exceed the local matter density (this is also called the "dominant energy condition").

In conclusion, these considerations point out that it is always possible to select fundamental length scales by considering masses and charges. Our aim is now to derive fundamental scales from a field theory and then connect them to astrophysical self-gravitating systems.

\section{THE LAGRANGIAN APPROACH}

The above relation can be derived from a Lagrangian formulation of extremal black holes. Basically there are two approaches, a non-supersymmetric one (see for example [14]) and a supersymmetric one (see for example [13]. In both approaches there are scalar fields (also called

\footnotetext{
${ }^{3} G=c=\hbar=\left(4 \pi \epsilon_{0}\right)^{-1}=1$
}

"moduli") coupled to gauge fields with dilaton like coupling. The scalar quantities are considered, at the beginning, without self-interacting potential.

For the non-supersymmetric case, let us consider a general situation with a 4-dimensional Lagrangian with $U(1)$ gauge fields and scalars with the "inclusion" of "axion" type couplings (see the last term) of the form

$$
\begin{aligned}
\mathcal{S}= & \frac{1}{k^{2}} \int d x^{4} \sqrt{-g}\left[R-2\left(\partial \varphi_{i}\right)^{2}+\right. \\
& -f_{a b}\left(\varphi_{i}\right) F_{\mu \nu}^{a} F^{b \mu \nu}+ \\
& \left.-\frac{1}{2} \tilde{f}_{a b}\left(\varphi_{i}\right) F_{\mu \nu}^{a} F_{\rho \sigma}^{b} \epsilon^{\mu \nu \rho \sigma}\right]
\end{aligned}
$$

which has $i=1, \ldots, N$ scalars with standard kinetic energy terms and $a=1, \ldots, M$ gauge fields ${ }^{4}$. It can be shown that the fields appearing in the metric obey equations of motion governed by an effective potential

$$
\begin{aligned}
V_{e f f}\left(\varphi_{i}\right)= & f^{a b}\left(Q_{e a}-\tilde{f}_{a c} Q_{m}^{c}\right)\left(Q_{e b}-\tilde{f}_{b d} Q_{m}^{d}\right) \\
& +\tilde{f}_{a b} Q_{m}^{a} Q_{m}^{b} .
\end{aligned}
$$

which is proportional to the energy density in the electromagnetic field. $Q_{e}$ and $Q_{m}$ are the electric and magnetic charges associated to the gauge field $F^{a}$, $f^{a b}$ being the inverse of the gauge coupling function $f_{a b}$. An effective potential can be derived also for the supersymmetric case. Before defining this, we observe that in both approaches, the so called "attractor mechanism" takes place once the following conditions are satisfied:

- the derivative of the potential has to be

$$
\partial_{i} V_{e f f}\left(\phi_{i 0}\right)=0
$$

where $\phi_{i 0}$ are the critical field values;

- the positive eigenvalue condition

$$
M_{i j}=\frac{1}{2} \partial_{i} \partial_{j} V_{e f f}\left(\phi_{i 0}\right)>0
$$

has to hold (see [15] for the simplest system described by one scalar and two gauge fields).

In the $N=2,4$ supersymmetric theories the above conditions are automatically satisfied. In fact, let us consider the effective potential for a black hole $V_{B H}$ with electric and magnetic charges in a supergravity $D=4, N=4$ theory (see [13])

\footnotetext{
4 A more general kinetic energy term for the scalars would arise from a Kahler supersymmetric theory. However, it is not necessary for our considerations here.
} 


$$
\begin{aligned}
V_{B H}\left(\phi, a, Q_{m}, Q_{e}\right)= & e^{2 \phi}\left[\left(a+i e^{-2 \phi}\right) Q_{m}-Q_{e}\right] \times \\
& {\left[\left(a-i e^{-2 \phi}\right) Q_{m}-Q_{e}\right] }
\end{aligned}
$$

where $\phi$ is the dilaton field, $a$ the axion one and only one component of the electromagnetic charges is considered for simplicity. Combining terms, one gets

$$
V_{B H}=e^{2 \phi}\left(Q_{e}-a Q_{m}\right)^{2}+e^{-2 \phi} Q_{m}^{2}
$$

On the other hand from conformal field theory (CFT) approach $^{5}$

$$
V_{e f f}^{C F T}=R_{c}^{2}\left(Q_{e}-\frac{\theta}{2 \pi} Q_{m}\right)^{2}+\frac{1}{R^{2}} Q_{m}^{2}
$$

where $R_{c}$ is the compactification radius of the scalar $\mathrm{Fu}-$ bini field [16]. If we equate $V_{\text {eff }}^{C F T}$ with $V_{B H}$ get the following identifications

$$
R_{c}^{2}=e^{2 \phi}, \quad \frac{\theta}{2 \pi}=a,
$$

where $\theta$ is a parameter (see [17]) and the dilaton axial scalar is

$$
s=a+i e^{-2 \phi}=\frac{\theta}{2 \pi}+\frac{i}{R_{c}^{2}} .
$$

Using the variable

$$
\zeta=\frac{\theta}{2 \pi}+i \frac{1}{g}
$$

we can see that

$$
V_{e f f}=g\left(Q_{e}-\frac{\theta}{2 \pi} Q_{m}\right)^{2}+\frac{1}{g} Q_{m}^{2}
$$

is invariant under the generalized duality transformations $\zeta \rightarrow \zeta+1$ and $\zeta=-\frac{1}{\zeta}[18]$.

Let us derive now the stationary (or critical) points of the above dynamics. We start with

$$
V_{e f f}=R_{c}^{2}\left(Q_{e}-\frac{\theta}{2 \pi} Q_{m}\right)^{2}+\frac{Q_{m}^{2}}{R_{c}^{2}} .
$$

Imposing $\frac{\partial V_{e f f}}{\partial R_{c}}=0$, we get ${ }^{6}$

$$
2 R_{c}\left(Q_{e}-\frac{\theta}{2 \pi} Q_{m}\right)^{2}-2 \frac{Q_{m}^{2}}{R c^{3}}=0
$$

\footnotetext{
5 The part of the interaction potential between charges dependent on the positions can be omitted due to the exat cancellation with its gravitational counterpart after imposing the no-net force condition [10 or its generalization which we will derive in (29).

${ }^{6}$ The derivatives of $V_{\text {eff }}$ are taken with respect to $R_{c}$ and not $\phi$ only for the sake of simplicity, since results 26) and (28) are not altered.
}

$$
\begin{gathered}
\Rightarrow R_{c}\left(Q_{e}-\frac{\theta}{2 \pi} Q_{m}\right)^{2}=\frac{Q_{m}^{2}}{R_{c}^{3}} \\
\Rightarrow R_{c}^{4}=\frac{Q_{m}^{2}}{Q_{e}-\frac{\theta}{2 \pi} Q_{m}} \\
\Rightarrow R_{H}^{2}=\frac{Q_{m}}{Q_{e}-\frac{\theta}{2 \pi} Q_{m}}=e^{2 \phi_{H}}
\end{gathered}
$$

where $R_{H}$ and $\phi_{H}$ indicate the corresponding values at the horizon of the black hole. Furthermore, we have

$$
\begin{aligned}
\frac{\partial^{2} V_{e f f}}{\partial R_{c}^{2}}= & 2\left(\frac{Q_{m}}{Q_{e}-\frac{\theta}{2 \pi} Q_{m}}\right)^{2}+\left.6 \frac{Q_{m}^{2}}{R_{c}^{4}}\right|_{R_{c}=\frac{Q_{m}}{Q_{e}-\frac{\theta}{2 \pi} Q_{m}}} \\
& =8\left(\frac{Q_{m}}{Q_{e}-\frac{\theta}{2 \pi} Q_{m}}\right)^{2}>0
\end{aligned}
$$

This means that the two conditions for the attractor mechanism are satisfied and the mass $M$ and charges $Q_{e}, Q_{m}$ of the extremal black holes saturate the BPS bound,

$$
M^{2}=e^{2 \phi_{H}}\left(Q_{e}-\frac{\theta}{2 \pi} Q_{m}\right)^{2}+e^{-2 \phi_{H}} Q_{m}^{2},
$$

or

$$
M^{2}=R_{H}^{2}\left(Q_{e}-\frac{\theta}{2 \pi} Q_{m}\right)^{2}+\frac{Q_{m}^{2}}{R_{H}^{2}},
$$

that generalize the above Eq.(10). After substituting $R_{H}^{2}=\frac{Q_{m}^{2}}{Q_{e}-\frac{\theta}{2 \pi} Q_{m}}$, we get

$$
M^{2}=2\left(Q_{e}-\frac{\theta}{2 \pi} Q_{m}\right) Q_{m}
$$

Let us now briefly comment on the above relations (22), (26), (28), (30)

- In the metric approach (see Sec II), the relation (28) is a consequence of $g_{00}=0$, that is we can say that the condition $g_{00}=0$ points out a phase transition, that is charged black holes are forming with mass $M$ and charges $Q_{e}, Q_{m}$ obeying the relation (28). We can imagine that, at its very beginning, the Universe was constituted by these primordial and "quantum" black holes with masses $M_{i}=M$, charges, $Q_{e}^{i}=Q_{e}$ and $Q_{m}^{i}=Q_{m}$ whose stability condition reads as

$$
\begin{aligned}
\sum_{i, j} M_{i} M_{j}= & \sum_{i, j} e^{2 \phi}\left(Q_{e}^{i}-\frac{\theta}{2 \pi} Q_{m}^{i}\right) \times \\
& \left(Q_{e}^{j}-\frac{\theta}{2 \pi} Q_{m}^{j}\right)+\left[e^{-2 \phi} Q_{m}^{i} Q_{m}^{j}\right],
\end{aligned}
$$


that is, using $M_{i}=M_{j}=M, Q_{e}^{i}=Q_{e}^{j}=Q_{e}$ and $Q_{m}^{i}=Q_{m}^{j}=Q_{m}$ as in Eq. (28). We can interpret the relation (26) as the compactification radius of the Fubini scalar field [16] describing the "correlations" of the black holes. Such scalar fields can be interpreted as the primary fields of the conformal field theory (CFT) describing the phase transition points or the "critical" points. Notice that the same relation (26) is realized in the CFT description of a quantum Hall fluid between the Fubini scalar field compactification radius and the electric and magnetic charges of the relavant excitations of the Hall fluid [19].

We have now all the ingredients to fix fundamental characteristic lengths from quantum black holes and then derive astrophysical sizes.

\section{QUANTUM BLACK HOLES TO FIX THE CHARACTERISTIC LENGTHS}

Without loosing any generality, let us consider the axion field $a=\frac{\theta}{2 \pi}=0$ and write

$$
M^{2}=2 Q_{e} Q_{m}
$$

In order to consider such black holes as quantum objects, we use the Dirac quantization condition [20]

$$
2 Q_{e} Q_{m}=n \hbar c
$$

with $n$ an integer $>0^{7}$. Substituting into the previous relation, one gets

$$
G M^{2}=n \hbar c
$$

For $n=1$, we get the lowest mass allowed for a quantum black hole (that we consider a primordial black hole):

$$
M_{B H}=\sqrt{\frac{\hbar c}{G}}=M_{\text {Planck }}
$$

Let us comment on this result. At very beginning $(t \simeq$ $10^{-43} \mathrm{sec}$ ) and at the "Planck" temperature, charged black holes were formed and constituted all the Universe mass. Thanks to the Dirac quantization condition (33), relation (34) appears to be very general due to the precence of the factor $n \hbar$, describing the angular momentum of the mass $M$. It is easy to show that such a quantization relation is valid for any given astrophysical mass by considering the ratio

$$
n_{\text {astro }}=\frac{r_{S-\text { astro }}}{2 \lambda_{\text {Compton-astro }}} .
$$

\footnotetext{
${ }^{7}$ Notice that standard units have been introduced here.
}

where we have assumed the Schwarzschild radius and the Compton length for any astrophysical structure We can check the scaling hypotesis in a few steps. We have

$$
G M_{\text {Planck }}^{2}=n_{\text {Planck }} \hbar c,
$$

where $n_{\text {Planck }}=1$ and

$$
G M_{u}^{2}=n_{u} \hbar c
$$

where $M_{u}$ is the whole Universe mass. Taking their ratio, one gets

$$
\frac{n_{u}}{n_{\text {Planck }}}=\left(\frac{M_{u}}{M_{\text {Planck }}}\right)^{2}
$$

By using $M_{u}=n_{p} m_{p}$ with $m_{p}=10^{-27} \mathrm{Kg}$, the proton mass and $n_{p}=10^{78}$, the number of protons in the Universe, we get

$$
\Rightarrow n_{u}=\left(10^{60}\right)^{2}=10^{120},
$$

That is the quantum relation (34) applies, in principle, to small scale structures (primordial Black Holes) up to the whole Universe, with 120 orders of magnitude between them, just the order of magnitude between the value of the cosmological constant $\Lambda$ at Planck scales and its present value ( 21] ).

We can rewrite relation (34) in another interesting form, using $n \hbar=J$, the angular momentum of the mass $M$ as

$$
J=\frac{G}{c} M^{2}
$$

Notice the strong resemblance with the corresponding relation for string states [22]. In order to show how such a scaling relation applies to any self-gravitating system, relation (41) can be rewritten in a more suitable form as

$$
J=\left(\frac{G M^{2}}{R}\right)\left(\frac{R}{c}\right)=E T,
$$

where $E$ is the characteristic gravitational energy of the astrophysical structure of "size" $R$ and $T$ is the characteristic crossing time for a light beam. Relation (41) is the starting point for the discussion in Sec. VII.

\section{THE EMERGENCE OF CHARACTERISTIC INTERACTION LENGTHS}

The above discussion shows that every unification scheme as superstrings, supergravity or grand unified theories, gives effective actions where non-minimal couplings to the geometry or higher order terms in the curvature invariants come out (an example is the above axion coupling). Such contributions are due to one-loop or higher-loop corrections in the high curvature regimes 
near the full (not yet available) quantum gravity regime [23]. However, in the weak-limit approximation, all these theories should be expected to reproduce the Einstein General Relativity which, in any case, is experimentally tested only in this limit 24].

This fact is matter of debate since most of relativistic theories do not reproduce Einstein results at the Newtonian approximation but, in some sense, generalize them [7] giving Yukawa-like corrections to the Newtonian potential which could have interesting physical consequences.

For example, some authors have shown that a conformal theory of gravity is nothing else but a fourth-order theory containing such terms in the Newtonian limit and, by invoking these results, it could be possible to explain the missing matter problem "without" dark matter [25].

A general effective theory of gravity coming from the renormalization of quantum fields on curved space-time can have an effective action of the form

$$
\begin{aligned}
\mathcal{A}=\int \quad & d^{4} x \sqrt{-g}\left[F\left(R, \square R, \square^{2} R, . . \square^{k} R, \phi\right)+\right. \\
& \left.-\frac{\epsilon}{2} g^{\mu \nu} \phi_{; \mu} \phi_{; \nu}+\mathcal{L}_{m}\right],
\end{aligned}
$$

where $F$ is an unspecified function of curvature invariants $R$ and $\square R$ and of scalar fields $\phi$. The term $\mathcal{L}_{m}$ is the minimally coupled ordinary matter contribution. Actually, more complicated invariants like $R_{\mu \nu} R^{\mu \nu}, R_{\mu \nu \alpha \beta} R^{\mu \nu \alpha \beta}$, $C_{\mu \nu \alpha \beta} C^{\mu \nu \alpha \beta}$ are also possible but we do not need them for the present purposes.

The weak field limit of theories of the form lead to gravitational potentials of the form [10, 26 28]

$$
V(r)=-\frac{G_{\infty} m}{r}\left[1+\sum_{k=1}^{n} \alpha_{k} e^{-r / \lambda_{k}}\right],
$$

where $G_{\infty}$ is the value of the gravitational constant as measured at infinity, $\lambda_{k}$ is the interaction length of the $k$-th component of non-Newtonian corrections. The amplitude $\alpha_{k}$ of each component is normalized to the standard Newtonian term (see [24, 29] for further details). The discussion involves also the variation of the gravitational coupling. As an example, let us take into account only the first term of the series in (44) which is usually considered the leading term (this choice is not sufficient if other corrections are needed). We have

$$
V(r)=-\frac{G_{\infty} m}{r}\left[1+\alpha_{1} e^{-r / \lambda_{1}}\right] .
$$

The effect of non-Newtonian term can be parameterized by $\left(\alpha_{1} \lambda_{1}\right)$. For large distances, at which $r \gg \lambda_{1}$, the exponential term vanishes and the gravitational coupling is $G_{\infty}$. If $r \ll \lambda_{1}$, the exponential becomes unity and, by differentiating, we get

$$
G_{l a b}=G_{\infty}\left[1+\alpha_{1}\left(1+\frac{r}{\lambda_{1}}\right) e^{-r / \lambda_{1}}\right]
$$

where $G_{l a b}=6.67 \times 10^{-8} \mathrm{~g}^{-1} \mathrm{~cm}^{3} \mathrm{~s}^{-2}$ is the usual Newton constant measured by Cavendish-like experiments. Of course, $G_{\infty}$ and $G_{l a b}$ coincide in standard gravity. It is worthwhile to note that, asymptotically, the inverse square law holds but the measured coupling constant differs by a factor $\left(1+\alpha_{1}\right)$. In general, any exponential correction introduces a characteristic length that acts at a certain scale for the self-gravitating systems. For a discussion and applications to the large scale structure, see [10].

This approach has been pursued by some authors who tested non-Newtonian corrections by ground-based experiments using totally different approachess [30 32]. The general outcome of these experiments, even retaining only the term $k=1$, is that a "geophysical window" between the laboratory and the astronomical results has to be taken into account. In fact, the range

$$
\left|\alpha_{1}\right| \sim 10^{-2}, \quad \lambda_{1} \sim 10^{2} \div 10^{3} \mathrm{~m},
$$

is not excluded at all. The sign of $\alpha_{1}$ tells us if corrections are attractive or repulsive. Another interesting suggestion has been given by Fujii [33], which proposed that the exponential deviation from the Newtonian standard potential (the "fifth force") could arise from the microscopic interaction which couples to nuclear isospin and baryon number.

The astrophysical counterpart of these non-Newtonian corrections seemed ruled out till some years ago due to the fact that experimental tests of general relativity predict "exactly" the Newtonian potential in the weak energy limit, "inside" the Solar System. Recently, as we said above, indications of an anomalous, longrange acceleration revealed from the data analysis of Pioneer 10/11, Galileo, and Ulysses spacecrafts makes these Yukawa-like corrections come into play [34]. Besides, reproduced the flat rotation curves of spiral galaxies can be reproduced by assuming [35]

$$
\alpha_{1}=-0.92, \quad \lambda_{1} \sim 40 \mathrm{kpc} .
$$

The main hypothesis is that the additional gravitational interaction is carried by an ultra-soft vector boson whose range of mass is $m_{0} \sim 10^{-27} \div 10^{-28} \mathrm{eV}$. The action of this boson becomes efficient at galactic scales without the request of enormous amounts of dark matter to stabilize the systems.

On the other hand, by asking for a characteristic length emerging from the standard theory of cosmological perturbation, it is possible to explain the observed segregation of hot stellar systems in the so called fundamental plane of galaxies ("ordinary" and "bright" galaxies) [36]. In that case, the length is the "Jeans length" of the protogalaxy $(\lambda \sim 3 \div 10 \mathrm{kpc})$ and, due to this characteristic size, a Yukawa correction was found in the gravitational potential with a characteristic interaction "length" of the same order of magnitude of that proposed by Sanders.

The emergence of characteristic lengths could be addressed by the above very fundamental approach capable 
of giving rise, in principle, to all self-gravitating systems. Our guess is that such lengths give rise to non-Newtonian corrections in the gravitational potential and then the aggregation of systems results extremely natural without the "ad hoc" introduction of dark matter constituents.

In the next section, we will discuss what we intend for the characteristic size of a self-gravitating system and then how they can be achieved starting from primordial quantum black holes in the framework of conformal theories.

\section{ASTROPHYSICAL SELF-GRAVITATING SYSTEMS}

In general, the concept of "size" of a self-gravitating system is not well-based since, in several cases, the boundary cannot be univocally defined. Let us briefly define globular clusters, galaxies, clusters and superclusters of galaxies considering their typical lengths and masses. This point is crucial since we are taking into account only systems where gravity is the only overall interaction acting between the components. In this sense, a star is not a purely self-gravitating system since, inside it, gravity is balanced by the pressure due to electromagnetic and nuclear interactions. However, we can take into account stars as granular constituents of globular clusters and galaxies and define a typical interaction length as the "size" of a planetary system around a star.

A globular cluster is a very compact self-gravitating stellar system whose typical radius is $R_{g c} \sim 10 \mathrm{pc}$. It contains up to $10^{6}$ stars $\left(M_{g c} \sim 10^{6} M_{\odot}\right)$ and is assumed completely virialized due to collisional interactions between stars.

On the other hand, a galaxy is a collisionless, diffuse gravitating system without an effective boundary. Astronomers define operative characteristic sizes as the effective radius $R_{e}$ which is the radius of the isophote containing half of the total luminosity, or the tidal radius $R_{t}$ corresponding to the distance from the center where the density drops to zero [37, 38]. Other definitions are possible by using photometry or kinematics but, assuming as a typical interaction size a length $R_{g} \sim 1 \div 10$ $\mathrm{kpc}$ is quite reasonable from dwarf to giant galaxies. It is important to point out that several authors assume that the halo of giant galaxies can extend as far as 100 $\mathrm{kpc}$ from the center taking into account the dark matter component. Here we do not assume any dark matter hypothesis and do not want to enter into details of galactic dynamics and morphology. For example, Milky Way, a typical spiral galaxy, has an observed disk scale length $R_{d} \simeq 3.5 \pm 0.5 \mathrm{kpc}$ while kinematics of globular clusters and $21 \mathrm{~cm}$-radio observations of neutral hydrogen give a maximal halo extension of $20 \div 30 \mathrm{kpc}$. For our purposes, assuming $10 \mathrm{kpc}$ as a characteristic size, with a possible error of an order of magnitude, is a good number. Typical masses are $M_{g} \sim 10^{10 \div 12} M_{\odot}$ for giant galaxies and $M_{g} \sim 10^{8 \div 9} M_{\odot}$ for dwarf galaxies.
As a cluster of galaxies, following Abell [39], we define a self-gravitating system whose granular components are galaxies with a typical radius $R_{c g}=R_{a} \simeq 1.5 h^{-1} \mathrm{Mpc}$ (the Abell radius) and a typical mass $M_{c g} \sim 10^{15} h^{-1} M_{\odot}$ for rich clusters, where $h$ is the dimensionless Hubble constant whose value is in the range $0.5<h<1$ [40].

Finally, a super-cluster is a self-gravitating system of clusters of galaxies whose typical size is $R_{s c} \sim 10 \div$ $100 h^{-1} \mathrm{Mpc}$ and typical mass is $M_{s c} \sim 10^{15 \div 17} h^{-1} M_{\odot}$.

Groups of galaxies are systems containing $10 \div 20$ galaxies, as our Local Group, but there are no evidences that they could be considered self-gravitating systems and, in any case, they are always part of more extended cluster of galaxies (in the case of Local Group, it is a part of the Virgo Cluster).

The main difference between a globular cluster and the other systems is that the former is a collisional system while the others are collisionless. This fact implies a completely different dynamical treatment [37].

The properties of these self-gravitating systems can be deduced by assuming them to be relaxed and virialized systems where gravity is the only overall interaction [37]. This assumption is, some times, not completely justified. In fact, we have to keep in mind that these systems undergo environmental effects, being never completely isolated; they always belong to larger gravitationally bound systems and the observational times are so short that the overall dynamics can only be extrapolated [37, 38].

Furthermore, as we said, the dynamics of astrophysical systems have to be related to cosmological evolution so that, in today observed dynamics, some quantum signature of primordial quantum perturbations should be present [2].

However the main difficulty, is to provide a physical route connecting the sizes of astrophysical structures with the extremely small numbers of quantum mechanics.

As a first step, we can build a model of the above structures composed of self-gravitating microscopic constituents (nucleons) which undergo quantum fluctuations coming from a very fundamental mechanism.

We introduce as the only observational input the number of nucleons contained. The characteristic dimension of such a model, as supposed above, is a functions of the microscopic nucleon scales (the Compton wavelength of a nucleon $\lambda_{c} \sim 10^{-13} \mathrm{~cm}$ ) and of the number of microscopic constituents.

The result is that the characteristic radii so deduced, numerically, coincide with those observed considering the usual gravitational constituents (stars for globular clusters and galaxies or galaxies themselves for clusters and super-clusters of galaxies, not nucleons).

Besides, we obtain a scaling relation between the units of length and action of the granular gravitational components, ranging from nucleons up to stars and galaxies.

On the other hand, the characteristic dimensions of astrophysical structures appear to be independent of the scale of the constituents considered. It is only needed 
that they depend on a minimal scale of length, which is, in order of magnitude, the Compton wavelength of a nucleon naturally deduced from the Planck length (see the above discussion).

These results suggest a macroscopic quantum coherence for large scale gravitational systems.

Furthermore, the emergence of these characteristic scales could have a dynamical counterpart in the Yukawa corrections of gravitational potential as in Eq.(44).

\section{SELF-GRAVITATING SYSTEMS FROM A CHARACTERISTIC FUNDAMENTAL LENGTH}

We want to show now that any self-gravitating system (from a star up to the whole Universe) can be achieved by quantum black holes defining a characteristic fundamental length

We have to consider the total action for the bound system with a very large number $N$ of constituents. The working hypothesis is that such an action can be achieved from the Planck constant. Let $E$ be the total energy. Let $\mathcal{T}$ be the characteristic global time of the system (e.g. the time in which a particle crosses the system, or the time in which the system evolves and becomes relaxed or virialized). By these two quantities, we get

$$
A \cong E \mathcal{T},
$$

which is the total action of the system (see also Eq. (42) to see how the heuristic following discussion can be related to fundamental arguments). The system could undergo a time-statistical fluctuation, so that the characteristic time $\tau$ for the stochastic motion per particle is

$$
\tau \cong \frac{\mathcal{T}}{\sqrt{N}} .
$$

We can then define an energy per granular component

$$
\epsilon \cong \frac{E}{N},
$$

so that the characteristic (minimal) unit of action $\alpha=$ $\epsilon \tau$ per granular component is expressed by the scaling relation

$$
\alpha=\epsilon \tau \cong \frac{A}{N^{3 / 2}} .
$$

This general considerations become physically consistent if the above fundamental theory is taken into account. Let us now consider the observational data for the above self-gravitating systems, in order of magnitude. Since we are taking into account virialized systems, we can assume

$$
2 E_{k}+U=0,
$$

where $E_{k}$ is the kinetic energy and $U$ the gravitational energy. The total energy $E$ then is

$$
E \simeq E_{k} \simeq N M v^{2}
$$

where $N$ is the typical number of granular components (e.g. stars in a galaxy or in a globular cluster, or galaxies in a cluster or super-cluster of galaxies; $M$ is the typical mass (e.g. $1 M_{\odot}$ for a Main Sequence star or $10^{10 \div 11} M_{\odot}$ for a galaxy like Milky Way); $v$ is a characteristic typical velocity which we choose to be the circular speed of the stars in the disk of galaxies $\left(\simeq 10^{7 \div 8} \mathrm{~cm} / \mathrm{sec}\right)$ or the velocity dispersion of the galaxies inside a cluster $\left(\simeq 10^{8 \div 9} \mathrm{~cm} / \mathrm{sec}\right)$. All these numbers are quite accurately measured by the methods of stellar kinematics, stellar statistics and photometry [37]. All the above quantities entering into the definitions of the energy, time and action scales are quantities coming from observations. In particular, nowhere we introduce the characteristic radius $R$ of the structures since this is what we wish to predict in the framework of our approach.

Let us take into account a galaxy. The energy per unit of mass is of the order $10^{15}(\mathrm{~cm} / \mathrm{sec})^{2}$, while the period of a galactic rotation, which can be assumed as the characteristic global time, is of the order [37]

$$
\mathcal{T}_{\text {rot }} \cong 10^{15} \mathrm{sec},
$$

and finally the total mass of a typical galaxy is of the order 37

$$
M_{g}=N_{s}^{(g)} M_{s} \cong 10^{44} \mathrm{gr} .
$$

From Eq.(49), combining these numbers, we get the typical action

$$
A \cong 10^{74} \mathrm{erg} \mathrm{sec} .
$$

The typical number of nucleons in a galaxy is 37]

$$
N_{p}^{(g)} \cong 10^{68} .
$$

Inserting these numbers in Eq.(52) we see that, up to an order of magnitude, the characteristic unit of action $\alpha$ of a galaxy, considered as an aggregate of nucleons, is of the order of the Planck action constant, $h \sim 10^{-27} \mathrm{erg}$ sec. It is interesting to notice that such a result is in full agreement with the quantization relation (34). In fact using in (34)

$$
M_{g}=N_{p}^{(g)} m_{p}=10^{68} \times 10^{-24} g=10^{44} g,
$$

we get

$$
n_{g}=\frac{G\left[N_{p}^{(g)} m_{p}\right]^{2}}{\hbar c}=10^{98}
$$


to be compared with

$$
\left(N_{p}^{(g)}\right)^{3 / 2}=10^{102}
$$

see Eqs. (52) and (58). That is the two results fully agree, within a few orders of magnitude allowed from statistical fluctuation, due for example for the presence of a "small" amount $(\simeq 2 \%)$ of heavy elements in the Universe (that is elements heavier than cosmological helium which could give rise to fluctuations in the nucleosynthesis [41]). It is worth stressing that also if dark matter is considered, the result does not change dramatically since the mass to luminosity ratio is of the order $10 \div 100$.

As a further step, we note that Eq.(52), together with the numerical result $\alpha \cong h$, can be re-formulated as a scaling relation for the mean action per microscopic component $a \equiv A / N$, that is

$$
a \cong h \sqrt{N} .
$$

We can then deduce that the fluctuative factor $\sqrt{N}$ provides the rescaling coefficient from the microscopic fundamental scales to the characteristic macroscopic dimensions. Let us now take into account the lengths. Given the nucleons as the basic microscopic constituents in our model, the natural quantum unit of length associated to each single constituent is the Compton wavelength $\lambda_{c}=h / m c$, with $c$ the velocity of light, and $m \cong m_{p} \cong 10^{-24} \mathrm{gr}$, the proton mass. In analogy with Eq.(62), we have, in general,

$$
R \cong \lambda_{c} \sqrt{N} .
$$

For a galaxy, with $N$ given by Eq.(58), we obtain

$$
R_{g} \cong 10^{21} \div 10^{22} \mathrm{~cm} \simeq 1 \div 10 \mathrm{kpc}
$$

which as we said above is a length of a galaxy. In particular, the numerical agreement of Eq.(63) with the observed galactic radii, is interesting, independently of the present derivation, since it links the scale of a large structure like a galaxy to the Compton wavelength of the elementary constituents (the nucleons) and to the total number of such constituents. It is worth noticing that, for typical galaxies, $R_{g}$ is the characteristic dimension where their rotation curve can be assumed flat [37] and where the halo and the disk stabilize each other.

The validity of Eq. (63) is not restricted to the galaxies, but provides the correct order of magnitude of the observed radii also if one considers the other structures which we mentioned above.

In the case of globular clusters, considering the suitable $N$ (which we easily deduce by the number of stars which constitute them, i.e. $10^{6}$ ), we get

$$
R_{g c} \cong 10^{18} \div 10^{19} \mathrm{~cm} \simeq 1 \div 10 \mathrm{pc} .
$$

For clusters of galaxies we obtain $R_{c g} \cong 1 \mathrm{Mpc}$ and for super-clusters $R_{s g} \cong 10 \div 100 \mathrm{Mpc}$.

The discussion can be extended to the whole Universe, and to other astrophysical objects, such as planetary systems (like the Solar System), provided one inserts in Eq.(63) the correct value of the number of nucleons $N$ contained in such structures.

These findings indicate that the quantum parameter $\lambda_{c}$ and the number of nucleonic constituents $N$, determine the observed astrophysical and cosmological dimensions.

The crucial objection to these results would be that stars or single galaxies, rather than nucleons, are the natural candidates as elementary gravitational constituents of a typical galaxy or a typical cluster of galaxies.

This apparent difficulty can be solved deriving a simple scaling law, which holds true at any scale. Let us take into account, for example, the number of stars $N_{s}^{(g)}$ contained in a typical galaxy, and the number $N_{n}^{(s)}$ of nucleons in a star. We can then obviously write, for the total number of nucleons in a typical galaxy

$$
N \cong N_{s}^{(g)} N_{n}^{(s)} .
$$

By Eq.(66), we can write Eq.(63) as

$$
R_{g} \cong \lambda_{s} \sqrt{N_{s}^{(g)}}
$$

where

$$
\lambda_{s} \equiv \frac{A_{s}}{M_{s} c}, \quad A_{s} \equiv h\left[N_{n}^{(s)}\right]^{3 / 2}, \quad M_{s} \equiv m N_{n}^{(s)} .
$$

Here, as above, $M_{s}$ is the total mass of a star, while the quantity $A_{s}$ is the characteristic unit of action of a star in the framework of our model, taking the stars as the elementary constituents of a typical galaxy. Inserting the numerical values [37 $N_{n}^{(s)} \cong 10^{57}, N_{s}^{(g)} \cong 10^{10} \div 10^{12}$, we obtain

$$
\lambda_{s} \cong 10^{13} \div 10^{15} \mathrm{~cm},
$$

which agrees with the typical range of interaction of a star (e.g. that of the Solar System), while for $R_{g}$ we obviously obtain again the value (64).

Therefore, Eqs.(63) and (67) show that we can derive the observed galactic radius $R_{g}$ either by considering a galaxy as a gas of $N$ nucleons with the fluctuation (50) defined with respect to $N$, or by considering, as usual, a typical galaxy as a gas of stars and assuming the fluctuative ansatz (50) rescaled with respect to the number of stars $N_{s}^{(g)}$.

The reason for the validity of this relation (which, in principle, holds on any scale) relies on the existence of a minimal scale of action which is needed for mechanical stability. In fact, the numerical value of the unit of 
action $A_{s}$ defined in Eq. 68 is $\cong 10^{58}$ erg s and thus coincides, in order of magnitude, with the total action for a typical star. Thus the rescaling relations (52) and (63) hold true also for a star, and $\lambda_{s}$ appears as the effective macroscopic "Compton wavelength" of a star. However $\lambda_{s}$ is the typical range of interaction also in the case of a globular cluster giving $R_{g c} \cong \lambda_{s} \sqrt{N_{s}^{(g c)}} \sim 1 \div 10 \mathrm{pc}$.

Immediately we derive $\lambda_{g}$ as the range of interaction for galaxies considered as granular constituents of clusters and super-clusters. Analogously, we have

$$
\lambda_{g} \equiv \frac{A_{g}}{M_{g} c}, \quad A_{g} \equiv h\left[N_{n}^{(g)}\right]^{3 / 2}, \quad M_{g} \equiv m N_{n}^{(g)}
$$

and then $\lambda_{g} \cong 10 \div 100 \mathrm{kpc}$. In this case, we can hierarchically consider a cluster or a super-cluster of galaxies as a gas of nucleons, stars or galaxies. It is interesting to note that $\lambda_{g}$ is the observed typical separation length between galaxies in a cluster.

At this point, it is straightforward the connection to the non-Newtonian gravitational potential (44). As we discussed above, the Yukawa corrections have to be reconducted to the emergence of typical scales for selfgravitating systems. For example, as discussed in 35. and in 42], by the interaction ranges of some vector bosons, it is possible to explain the flat rotation curves of spiral galaxies without asking for large amounts of dark matter. Yukawa corrections naturally emerge in relation to these interaction ranges. The main shortcoming of their approach is that, till now, no ultra-light vector boson has been detected and the requested interaction lengths $\lambda \sim 10 \mathrm{kpc}$ are very hard to justify.

By our hypothesis, as we discussed above, $\lambda_{g} \sim 10 \mathrm{kpc}$ naturally emerges by taking into account the granular components of a galaxy. Using, Eq.45 where we assume $\lambda_{1}=\lambda_{g}$, the arguments in [35] and in [42] are easily recovered.

Besides, the anomalous, long-range acceleration reported in 34] immediately outside the Solar System, could be explained considering a Yukawa correction in the Newtonian potential related to a length as $\lambda_{s}$ which can be considered as the typical range of interaction of a star as the Sun (a system with gravitationally bound planets).

\section{DISCUSSION AND CONCLUSIONS}

In this paper, we have discussed the possibility that the characteristic sizes of astrophysical self-gravitating systems could be deduced by scaling laws relating the observed macroscopic dimensions to the microscopic funda- mental scales originated by the primordial quantum black holes. The net effects at macroscopic scales are Yukawalike corrections to the Newtonian potential which become relevant in the range $r \sim \lambda$. This fact could be connected to the well-known absence of screening mechanisms for gravity [43].

Before drawing the conclusions, we have to discuss the scales of action involved. Another link between the quantum unit of action $h$ and the cosmological scales is provided by the so-called Eddington-Weinberg relation $h \cong G^{1 / 2} m^{3 / 2} R^{1 / 2}$, where $G$ is the Newton gravitational constant, $m$ is the mass of the nucleon, and $R$ is the radius of the Universe. If one takes for $R$ the various definitions of cosmological radius (Hubble radius, causal radius, or last scattering radius) 40] which range from $R=10^{26} \mathrm{~cm}$ to $R=10^{30} \mathrm{~cm}$, one obtains a value for the unit of action $\alpha$ ranging from $10^{-26}$ erg sec to $10^{-27}$ erg sec which is usually assumed to coincide with the Planck constant $h$ in order of magnitude [44, 45].

A similar relation can be deduced also for the selfgravitating structures which we have discussed, if one inserts in the equations for $A$ and $\alpha$ the gravitational energy $U(R)$ and a characteristic gravitational time $\mathcal{T}$ needed for the relaxation of the system. In this case, a sort of Eddington-Weinberg relation can be derived and appears to hold also for galaxies, and other large scale structures as clusters and super-clusters of galaxies yielding a microscopic unit of action of the order of $10^{-(27 \div 28)}$ erg sec.

The numerical coincidence up to two orders of magnitude of the Eddington-Weinberg relations for the Universe and for large scale structures is not purely accidental if the quantum black holes are ruling the astrophysical structures. Therefore, as we have shown, what is really significant in our model, both on astrophysical and cosmological scales, are the micro/macro scaling relations and connectivity factors, that is Eqs.(50), (52), and (63), which hold true for all systems and are built starting from the basic assumption of nuclear granularity [5, $[6]$.

In conclusion, the typical hierarchical sizes of astrophysical structures could be explained by taking into account a very fundamental length giving rise to the typical interaction ranges of self-gravitating systems. Dynamics is implemented by a non-Newtonian gravitational potential where Yukawa corrections effectively act at that given typical scale. However, the value of the gravitational coupling is different at the various distances depending on the interaction ranges $\lambda_{c}, \lambda_{s}, \lambda_{g}$. Finally, we want to stress that no additional (unknown) dark matter particle has been introduced and the standard nucleons can completely account for sizes and stability of astrophysical structures.
[1] E. W. Kolb and M. S. Turner, The Early Universe Addison-Wesley, New York (1990).
[2] A. Sakharov, Zh. Eksp. Teor. Fiz. 49, 245 (1965); 
V. F. Mukhanov, H. A. Feldman and R. H. Brandenberger, Phys. Rep. 215, 203 (1992).

[3] V. de Lapparent, M. J. Geller and J. P. Huchra, Astrophys. Jour. 302, L1 (1986);

M. J. Geller and J. P. Hucra, Science 246, 897 (1989);

R. De Propris, M.M. Colless, S.P. Driver et al. MNRAS 342725 (2003);

D. Burkey, A.N. Taylor, MNRAS 347, 255 (2004).

[4] F. Calogero, Phys. Lett. A 228, 335 (1997).

[5] S. Capozziello and S. Funkhouser, Mod. Phys. Lett. A 24, 1121 (2009).

[6] S. Capozziello and S. Funkhouser, Mod. Phys. Lett. A 24, 1743 (2009).

[7] S. Nojiri and S.D. Odintsov, IJGMMP 4, 115 (2007); S. Capozziello and M. Francaviglia, Gen. Rel. Grav. 40, 357 (2008);

T. Sotiriou and V. Faraoni, Rev. Mod. Phys. 82, 451 (2010).

[8] S.S. McGaugh, Ap. J. Lett. 541, L33 (2000).

[9] P. de Bernardis, et al., Nature 404, 955 (2000); http://lambda.gsfc.nasa.gov/product/map/current/map http://www.sciops.esa.int/index.php?project=PLANCK

[10] S. Capozziello, V.F. Cardone, A. Troisi, MNRAS 375, 1423 (2007);

S. Capozziello, E. De Filippis, V. Salzano, MNRAS, 394, 947 (2009).

[11] S. Chandrasekhar, "The Mathematical Theory of Black Holes", Clarendon Press, Oxford Science Publications, Oxford (1983).

[12] S.L. Shapiro and S.A. Teukolsky, Phys. Rev. Lett. 66, 994 (1991).

[13] S. Ferrara, K. Hayakawa, A. Marrani, arXiv:0805.2498v2 [hep-th] .

[14] K. Goldstein, N. Iizuka, R. P. Jena, S. P. Trivedi, Phys.Rev. D 72, 124021 (2005).

[15] K. Goldstein, S. Kachru, S. Prakash, S. P. Trivedi, arXiv:0911.3586v3.

[16] S. Fubini, Mod.Phys.Lett. A 6, 347 (1991).

[17] G. 't Hooft, Nucl. Phys. 138, 1 (1978).

[18] J. L. Cardy, E. Rabinovici, Nucl. Phys. B 205 ,1 (1982).

[19] G. Cristofano, G. Maiella, R. Musto, F. Nicodemi, Mod.Phys.Lett. A 6, 1779 (1991);

G. Cristofano, D. Giuliano, G. Maiella, Phys Lett. B 504, 345 (2001).

[20] P.M.A. Dirac, Proc. Roy. Soc. A 133, 60, (1931).

[21] S. Weinberg, Gravitation and Cosmology, Wiley, New York (1972)

S. Weinberg, Rev. Mod. Phys. 61, 1 (1989).

[22] M.B. Green, J. H. Schwarz, E. Witten, Superstring The- ory, Cambridge Univ. Press, Cambridge (1987).

[23] I.L. Buchbinder, S.D. Odintsov, and I.L. Shapiro, Effective Action in Quantum Gravity, IOP Publishing Bristol (1992).

[24] C.M. Will, Theory and Experiments in Gravitational Physics, Cambridge Univ. Press, Cambridge (1993).

[25] P.D. Mannheim and D. Kazanas, Astroph. Jou. 342, 635 (1989).

O.V. Barabash and Yu. V. Shtanov, Phys. Rev. D60, 064008 (1999).

[26] K. S. Stelle, Gen. Rel. Grav. 9, 353 (1978).

[27] S. Capozziello, A. Stabile and A. Troisi, Phys. Rev. D 76, 104019 (2007).

[28] S. Capozziello, E. Elizalde, S. Nojiri, and S. D. Odintsov, Phys. Lett. B 671, 193 (2009).

[29] A.H. Cook in 300 Years of Gravitation, Eds. S. Hawking and W. Israel, Cambridge Univ. Press, Cambridge (1987).

[30] E. Fischbach, D. Sudarsky, A. Szafer, C. Talmadge, and S.H. Aroson, Phys. Rev. Lett. 56, 3 (1986) .

[31] C.C. Speake and T.J. Quinn, Phys. Rev. Lett. 61, 1340 (1988).

[32] D.H. Eckhardt, C. Jekeli, A.R. Lazarewicz, A.J. Romaides, Phys. Rev. Lett. 60, 2567 (1988).

[33] Y. Fujii, Phys. Lett. B 202246 (1988).

[34] J.D. Anderson et al., Phys. Rev. Lett. 81, 2858 (1998).

[35] R.H. Sanders, Astron. Astrophys. Rev. 2, 1 (1990).

[36] M. Capaccioli, S. Capozziello, G. Longo, and R. de Ritis, Astron. Nachr. 318, 1 (1997).

[37] J. Binney and S. Tremaine, Galactic Dynamics, Princeton University Press, Princeton (1987).

[38] B.A. Vorontsov-Vel'yaminov, Extragalactic Astronomy Harwood Academic Pub., London (1987).

[39] G.O. Abell, Astroph. Jou. S. 3, 211 (1958).

[40] P. J. E. Peebles, Principles of Physical Cosmology (Princeton University Press, Princeton (1993).

[41] S. Burles, K. M. Nollett, M. S. Turner, Phys. Rev. D 63, 063512 (2001).

[42] D. H. Eckhardt, Phys. Rev. D 48, 3762 (1993).

[43] O. Bertolami, J.M. Mourao, and J. Perez-Mercader, Phys. Lett. B 311, 27 (1993);

T. Goldman, J. Pérez-Mercader, F. Cooper, and M.M. Nieto, Phys. Lett. B 281, 219 (1992).

[44] J.D. Barrow and F.J. Tipler, The Anthropic Cosmological Principle (Oxford Univ. Press, Oxford (1986).

[45] S. Roy Statistical Geometry and Applications to Microphysics and Cosmology Kluwer Academic Pub., Dordrecht (1998). 